\title{
Calculation of the CIPW norm: New formulas
}

\author{
KAMAL L PRUSETH \\ Institute Instrumentation Centre, Indian Institute of Technology Roorkee, Roorkee 247 667, India.
}

\begin{abstract}
A completely new set of formulas, based on matrix algebra, has been suggested for the calculation of the CIPW norm for igneous rocks to achieve highly consistent and accurate norms. The suggested sequence of derivation of the normative minerals greatly deviates from the sequence followed in the classical scheme. The formulas are presented in a form convenient for error-free implementation in computer programs. Accurate formulas along with the use of variable molecular weights for $\mathrm{CaO}$ and $\mathrm{FeO}$; corrected formula weights for apatite, pyrite and fluorite; and suggested measures to avoid significant rounding off errors to achieve absolute match between the sum of the input weights of the oxides and the sum of the weights of the estimated normative minerals. Using an analogous procedure for determining the oxidation ratios of igneous rocks as used in the SINCLAS system of Verma et al $(2002,2003)$, the suggested calculation scheme exactly reproduces their results except for apatite for reasons explained in the text, but with a superior match between the totals for about 11,200 analyses representing rocks of a wide range of composition.
\end{abstract}

\section{Introduction}

The CIPW norm provides the basis for the comparison of aphanitic igneous rocks with their phaneritic counterparts. However, the main application of it is in the classification of glassy and very fine-grained volcanic rocks where classification from the evaluation of the mode is not possible (see Verma et al 2003 for a detailed discussion). Therefore, to achieve consistency in classification, a standard igneous norm calculation method is essential (Middlemost 1989). Now-a-days norms are usually calculated using digital computers. The classic norm calculation procedure (Cross et al 1902) and its later modifications (Washington 1917; Johannsen 1931; Hutchison 1974; Cox et al 1979; Le Maitre 1982; Ragland 1989; Rollinson 1993) however, are not specifically suitable for implementation on a computer leading to different methods of implementation by different programmers. Kelsey (1965) modified the portions used especially for the estimation of silica undersaturated minerals in the norm. These modifications however, are not universally adopted in the computer programs that are available for calculation of the norm. The SINCLAS implementation of Verma et al (2002, 2003) which is based and modified after Kelsey (1965) is the most reliable and consistent of all.

Additionally, there is no unique way of fixing the $\mathrm{FeO} / \mathrm{Fe}_{2} \mathrm{O}_{3}$ ratios (oxidation ratios) of igneous rocks which in turn, significantly change the nature and amount of the normative minerals of rocks (Middlemost 1989). Therefore no standardization is possible without evolving a universally agreed procedure to determine the oxidation ratio. Oxidation ratios obtained from chemical analysis either are not acceptable as most of the igneous rocks show higher $\mathrm{Fe}_{2} \mathrm{O}_{3}$ in their analysis because of the oxidation postdating their consolidation. Until a method is evolved to uniquely fix the oxidation ratio, standardization can only be achieved for the details of the calculation procedure. The SINCLAS system of Verma et al $(2002,2003)$ even offers three options on how to fix the $\mathrm{FeO} / \mathrm{Fe}_{2} \mathrm{O}_{3}$ ratios and involves an amount of subjectivity on the part of the user.

At the end of the calculation of norm for a rock it is customary to report the sum of weights of

Keywords. CIPW norm; igneous rock classification; oxidation ratio; standard igneous norm; normative minerals. 
Table 1. Composition and inverse composition matrices for the saturated normative minerals.

\begin{tabular}{lccccccc}
\hline & $\mathrm{C}$ & $\mathrm{Or}$ & $\mathrm{Ab}$ & $\mathrm{An}$ & $\mathrm{En}$ & $\mathrm{Fs}$ & $\mathrm{Hm}$ \\
\hline $\mathrm{Al}_{2} \mathrm{O}_{3}$ & 1 & 1 & 1 & 1 & 0 & 0 & 0 \\
$\mathrm{Fe}_{2} \mathrm{O}_{3}$ & 0 & 0 & 0 & 0 & 0 & 0 & 1 \\
$\mathrm{FeO}$ & 0 & 0 & 0 & 0 & 0 & 1 & 0 \\
$\mathrm{MgO}$ & 0 & 0 & 0 & 0 & 1 & 0 & 0 \\
$\mathrm{CaO}$ & 0 & 0 & 0 & 1 & 0 & 0 & 0 \\
$\mathrm{Na}{ }_{2} \mathrm{O}$ & 0 & 0 & 1 & 0 & 0 & 0 & 0 \\
$\mathrm{~K}_{2} \mathrm{O}$ & 0 & 1 & 0 & 0 & 0 & 0 & 0 \\
& $\mathrm{Al}_{2} \mathrm{O}_{3}$ & $\mathrm{Fe}_{2} \mathrm{O}_{3}$ & $\mathrm{FeO}$ & $\mathrm{MgO}$ & $\mathrm{CaO}$ & $\mathrm{Na}_{2} \mathrm{O}$ & $\mathrm{K}_{2} \mathrm{O}$ \\
\hline $\mathrm{C}$ & 1 & 0 & 0 & 0 & -1 & -1 & -1 \\
$\mathrm{Or}$ & 0 & 0 & 0 & 0 & 0 & 0 & 1 \\
$\mathrm{Ab}$ & 0 & 0 & 0 & 0 & 0 & 1 & 0 \\
$\mathrm{An}$ & 0 & 0 & 0 & 0 & 1 & 0 & 0 \\
$\mathrm{En}$ & 0 & 0 & 0 & 1 & 0 & 0 & 0 \\
$\mathrm{Fs}$ & 0 & 0 & 1 & 0 & 0 & 0 & 0 \\
$\mathrm{Hm}$ & 0 & 1 & 0 & 0 & 0 & 0 & 0 \\
\hline
\end{tabular}

the normative minerals and to compare this value with the sum of weights of the oxides obtained from chemical analysis. Le Maitre (1982) lays great emphasis on the necessity of match between the two up to at least the third place of decimal. Very often this practice offers a means of checking the accuracy of the norm calculation, but the mismatch is not always due to the inaccuracy in the values of the estimated norms. However, perfect match between the two totals imparts a sense of confidence to the calculation and a standard norm calculation procedure should be able to exactly account for the disagreement between the two sums.

It is also observed that not all the computer programs available for the calculation of norm, work satisfactorily over the whole range of composition of igneous rocks. This is probably due to the errorprone schemes and complex decision making steps involved in the calculation of the norms.

Here, a completely new set of formulas has been suggested, based on linear algebraic and mass balance approaches, which are easily understandable and will therefore, lead to less error-prone implementation on a computer. Extension of the classic scheme is also suggested to take care of extremely $\mathrm{SiO}_{2}$-poor rocks in which the deficiency in $\mathrm{SiO}_{2}$ cannot be completely compensated for even after converting all leucite to kaliophilite. Additionally, further causes of mismatch between the totals of oxides and normative minerals have been discussed, which when taken care of, achieve an absolute match between the two. The norm calculation steps have been presented in a manner so as to provide maximum convenience in implementing them on a computer and to avoid ambiguity as far as possible.

\section{The method}

The composition of the saturated normative minerals can be represented by a composition matrix as given in table 1 . The inverse composition matrix (table 1) can be multiplied on the left of a column matrix that contains the analysis of $\mathrm{Al}_{2} \mathrm{O}_{3}$, $\mathrm{Fe}_{2} \mathrm{O}_{3}, \mathrm{FeO}, \mathrm{MgO}, \mathrm{CaO}, \mathrm{Na}_{2} \mathrm{O}$ and $\mathrm{K}_{2} \mathrm{O}$ in terms of their molecular proportions obtained by dividing the weight percentages by their respective molecular weights. From the inspection of the inverse composition matrix, it is evident that the same can be written in terms of the following formulas to obtain the provisional saturated minerals.

$$
\begin{array}{ll}
\mathrm{Or}_{\mathrm{p}}=\mathrm{K}_{2} \mathrm{O}, & \mathrm{Ab}_{\mathrm{p}}=\mathrm{Na}_{2} \mathrm{O}, \\
\mathrm{An}_{\mathrm{p}}=\mathrm{CaO}, & \mathrm{En}_{\mathrm{p}}=\mathrm{MgO}, \\
\mathrm{Fs}_{\mathrm{p}}=\mathrm{FeO}, & \mathrm{Hm}_{\mathrm{p}}=\mathrm{Fe}_{2} \mathrm{O}_{3}, \\
\mathrm{C}_{\mathrm{p}}=\mathrm{Al}_{2} \mathrm{O}_{3}-\mathrm{CaO}-\mathrm{Na}_{2} \mathrm{O}-\mathrm{K}_{2} \mathrm{O}
\end{array}
$$

The mineral symbols are given in table 2 . The subscript $\mathrm{p}$ stands for 'provisional', and numerical subscripts whenever used for intermediate values.

If provisional corundum $\mathrm{C}_{\mathrm{p}}$ is negative, then some of the provisional minerals are broken down in specific order one by one to produce 
Table 2. List of oxides with their molecular weights (based on IUPAC Commission on Atomic Weights and Isotopic Abundances; Vocke 1999) and normative minerals and their formulas used in the calculation of the norm.

\begin{tabular}{|c|c|c|c|}
\hline $\mathrm{SiO}_{2}$ & 60.0843 & Quartz (q) & $\mathrm{SiO}_{2}$ \\
\hline $\mathrm{TiO}_{2}$ & 79.8658 & Corundum (c) & $\mathrm{Al}_{2} \mathrm{O}_{3}$ \\
\hline $\mathrm{Al}_{2} \mathrm{O}_{3}$ & 101.9613 & Zircon $(\mathrm{z})$ & $\mathrm{ZrO}_{2} \cdot \mathrm{SiO}_{2}$ \\
\hline $\mathrm{Fe}_{2} \mathrm{O}_{3}$ & 159.6882 & Orthoclase (or) & $\mathrm{K}_{2} \mathrm{O} \cdot \mathrm{Al}_{2} \mathrm{O}_{3} \cdot 6 \mathrm{SiO}_{2}$ \\
\hline $\mathrm{FeO}$ & 71.8444 & Albite (ab) & $\mathrm{Na}_{2} \mathrm{O} \cdot \mathrm{Al}_{2} \mathrm{O}_{3} \cdot 6 \mathrm{SiO}_{2}$ \\
\hline $\mathrm{MnO}$ & 70.93745 & Anorthite (an) & $\mathrm{CaO} \cdot \mathrm{Al}_{2} \mathrm{O}_{3} \cdot 2 \mathrm{SiO}_{2}$ \\
\hline $\mathrm{NiO}$ & 74.6928 & Leucite (lc) & $\mathrm{K}_{2} \mathrm{O} \cdot \mathrm{Al}_{2} \mathrm{O}_{3} \cdot 4 \mathrm{SiO}_{2}$ \\
\hline $\mathrm{MgO}$ & 40.3044 & Nepheline (ne) & $\mathrm{Na}_{2} \mathrm{O} \cdot \mathrm{Al}_{2} \mathrm{O}_{3} \cdot 2 \mathrm{SiO}_{2}$ \\
\hline $\mathrm{CaO}$ & 56.0774 & Kaliophilite (kp) & $\mathrm{K}_{2} \mathrm{O} \cdot \mathrm{Al}_{2} \mathrm{O}_{3} \cdot 2 \mathrm{SiO}_{2}$ \\
\hline $\mathrm{BaO}$ & 153.3264 & Acmite (ac) & $\mathrm{Na}_{2} \mathrm{O} \cdot \mathrm{Fe}_{2} \mathrm{O}_{3} \cdot 4 \mathrm{SiO}_{2}$ \\
\hline $\mathrm{SrO}$ & 103.6194 & Sodium metasilicate (ns) & $\mathrm{Na}_{2} \mathrm{O} \cdot \mathrm{SiO}_{2}$ \\
\hline $\mathrm{Na}_{2} \mathrm{O}$ & 61.97894 & Potassium metasilicate (ks) & $\mathrm{K}_{2} \mathrm{O} \cdot \mathrm{SiO}_{2}$ \\
\hline $\mathrm{K}_{2} \mathrm{O}$ & 94.196 & Diopside (di) & $\mathrm{CaO} \cdot(\mathrm{Mg}, \mathrm{Fe}) \mathrm{O} \cdot 2 \mathrm{SiO}$ \\
\hline $\mathrm{P}_{2} \mathrm{O}_{5}$ & 141.9445 & Wollastonite (wo) & $\mathrm{CaO} \cdot \mathrm{SiO}_{2}$ \\
\hline $\mathrm{S}$ & 32.066 & Hypersthene (hy) & $(\mathrm{Mg}, \mathrm{Fe}) \mathrm{O} \cdot \mathrm{SiO}_{2}$ \\
\hline $\mathrm{Cr}_{2} \mathrm{O}_{3}$ & 151.9904 & Olivine (ol) & $2(\mathrm{Mg}, \mathrm{Fe}) \mathrm{O} \cdot \mathrm{SiO}_{2}$ \\
\hline $\mathrm{F}$ & 18.9984 & Dicalcium silicate (cs) & $2 \mathrm{CaO} \cdot \mathrm{SiO}_{2}$ \\
\hline $\mathrm{CO}_{2}$ & 44.0095 & Magnetite (mt) & $\mathrm{FeO} \cdot \mathrm{Fe}_{2} \mathrm{O}_{3}$ \\
\hline \multirow[t]{10}{*}{$\mathrm{ZrO}_{2}$} & 123.2228 & Chromite $(\mathrm{cm})$ & $\mathrm{FeO} \cdot \mathrm{Cr}_{2} \mathrm{O}_{3}$ \\
\hline & & Ilmenite (il) & $\mathrm{FeO} \cdot \mathrm{TiO}_{2}$ \\
\hline & & Hematite $(\mathrm{hm})$ & $\mathrm{Fe}_{2} \mathrm{O}_{3}$ \\
\hline & & Titanite (tn) & $\mathrm{CaO} \cdot \mathrm{TiO}_{2} \cdot \mathrm{SiO}_{2}$ \\
\hline & & Perovskite (pf) & $\mathrm{CaO} \cdot \mathrm{TiO}_{2}$ \\
\hline & & Rutile (ru) & $\mathrm{TiO}_{2}$ \\
\hline & & Apatite (ap) & $9 \mathrm{CaO} \cdot 3 \mathrm{P}_{2} \mathrm{O}_{5} \cdot \mathrm{CaF}_{2}$ \\
\hline & & Fluorite (fr) & $\mathrm{CaF}_{2}$ \\
\hline & & Pyrite (py) & $\mathrm{FeS}_{2}$ \\
\hline & & Calcite (cc) & $\mathrm{CaO} \cdot \mathrm{CO}_{2}$ \\
\hline
\end{tabular}

$\mathrm{Al}_{2} \mathrm{O}_{3}$-undersaturated minerals until the deficiency in $\mathrm{Al}_{2} \mathrm{O}_{3}$ is resolved. For example, anorthite $\left(\mathrm{CaAl}_{2} \mathrm{Si}_{2} \mathrm{O}_{8}\right)$ can be partly or fully converted to wollastonite $\left(\mathrm{CaSiO}_{3}\right)$. An and Wo can be represented by the equations:

$$
\mathrm{An}=0 \mathrm{Al}_{2} \mathrm{O}_{3}+1 \mathrm{CaAl}_{2} \mathrm{Si}_{2} \mathrm{O}_{8}=0 \mathrm{C}_{\mathrm{p}}+1 \mathrm{An}_{\mathrm{p}}
$$

and

$$
\begin{aligned}
\text { Wo } & =1 \mathrm{CaAl}_{2} \mathrm{Si}_{2} \mathrm{O}_{8}-1 \mathrm{Al}_{2} \mathrm{O}_{3}-\mathrm{SiO}_{2} \\
& =1 \mathrm{An}_{\mathrm{p}}-1 \mathrm{C}_{\mathrm{p}}-1 \mathrm{Q}_{\mathrm{p}} .
\end{aligned}
$$

The composition and inverse composition matrices representing this breakdown reaction can be written as below. Note that $\mathrm{SiO}_{2}$ and $\mathrm{Q}$ are not represented in the composition matrix as it is intended here to adjust the $\mathrm{Al}_{2} \mathrm{O}_{3}$ component. The gain in $\mathrm{SiO}_{2}$ is finally taken into account in the estimate of quartz $(\mathrm{Q})$, after all other silicate phases have been estimated.

\begin{tabular}{l|cr} 
& $\mathrm{An}$ & Wo \\
\hline $\mathrm{C}_{\mathrm{p}}$ & 0 & -1 \\
$\mathrm{An}_{\mathrm{p}}$ & 1 & 1
\end{tabular}

\begin{tabular}{c|rc} 
& $\mathrm{C}_{\mathrm{p}}$ & $\mathrm{An}_{\mathrm{p}}$ \\
\hline $\mathrm{An}$ & 1 & 1 \\
$\mathrm{Wo}$ & -1 & 0
\end{tabular}

The new estimates of $\mathrm{An}$ and Wo are calculated according to the inverse matrix as $A n=C_{p}+A_{p}$ 
and $\mathrm{Wo}=-\mathrm{C}_{\mathrm{p}}$. Once the deficiency of $\mathrm{Al}_{2} \mathrm{O}_{3}$ is fully resolved, the amount of $\mathrm{SiO}_{2}$ consumed by the provisional minerals is calculated and if it is found greater than the $\mathrm{SiO}_{2}$ content of the rock whose norm is being calculated, $\mathrm{SiO}_{2}$-undersaturated phases are calculated sequentially until the deficiency is completely removed. For example, olivine $\left((\mathrm{Mg}, \mathrm{Fe})_{2} \mathrm{SiO}_{4}\right)$ is formed by breaking down provisional hypersthene $\left((\mathrm{Mg}, \mathrm{Fe}) \mathrm{SiO}_{3}\right)$ using the formulas, $\mathrm{Ol}=-\mathrm{Q}$ and $\mathrm{Hy}=2 \mathrm{Q}+\mathrm{Hy}_{\mathrm{p}}$ according to the matrices,

\begin{tabular}{l|rr} 
& $\mathrm{Ol}$ & $\mathrm{Hy}$ \\
\hline $\mathrm{Q}$ & -1 & 0 \\
$\mathrm{Hy}_{\mathrm{p}}$ & 2 & 1
\end{tabular}

\begin{tabular}{c|rc} 
& $\mathrm{Q}$ & $\mathrm{Hy}_{\mathrm{p}}$ \\
\hline $\mathrm{Ol}$ & -1 & 0 \\
$\mathrm{Hy}$ & 2 & 1
\end{tabular}

where, Q represents the deficiency of $\mathrm{SiO}_{2}$. If $\mathrm{Hy}$ is found to be negative after this step, further desilication reactions are required, for example,

$$
\begin{aligned}
& \mathrm{Ol}=0.5 \mathrm{Hy}_{\mathrm{p}} \\
& \mathrm{Ne}=-0.25 \mathrm{Q}-0.125 \mathrm{Hy}_{\mathrm{p}} \\
& \mathrm{Ab}=0.25 \mathrm{Q}+0.125 \mathrm{Hy}_{\mathrm{p}}+\mathrm{Ab}_{\mathrm{p}}
\end{aligned}
$$

for which the matrices are,

\begin{tabular}{l|rrr} 
& $\mathrm{Ol}$ & $\mathrm{Ne}$ & $\mathrm{Ab}$ \\
\hline $\mathrm{Q}$ & -1 & -4 & 0 \\
$\mathrm{Hy}_{\mathrm{p}}$ & 2 & 0 & 0 \\
$\mathrm{Ab}_{\mathrm{p}}$ & 0 & 1 & 1
\end{tabular}

\begin{tabular}{c|ccc} 
& $\mathrm{Q}$ & $\mathrm{Hy}_{\mathrm{p}}$ & $\mathrm{Ab}_{\mathrm{p}}$ \\
\hline $\mathrm{Ol}$ & 0 & 0.5 & 0 \\
$\mathrm{Ne}$ & -0.25 & -0.125 & 0 \\
$\mathrm{Ab}$ & 0.25 & 0.125 & 1
\end{tabular}.

Likewise, amounts of the other $\mathrm{SiO}_{2}$-undersaturated phases are determined. Finally, for extremely $\mathrm{SiO}_{2}$-undersaturated rocks one further step has been added in which anorthite is broken down to dicalcium silicate and corundum according to the composition and inverse composition matrices:

\begin{tabular}{l|lcc} 
& $\mathrm{Cs}$ & $\mathrm{C}$ & $\mathrm{An}$ \\
\hline $\mathrm{Q}$ & 0 & -1.5 & 0 \\
$\mathrm{Cs}_{\mathrm{p}}$ & 1 & -0.5 & 0 \\
$\mathrm{An}_{\mathrm{p}}$ & 0 & 1 & 1 \\
& $\mathrm{Q}$ & $\mathrm{Cs}_{\mathrm{p}}$ & $\mathrm{An}_{\mathrm{p}}$ \\
\hline $\mathrm{Cs}$ & $-1 / 3$ & 1 & 0 \\
$\mathrm{C}$ & $-2 / 3$ & 0 & 0 \\
$\mathrm{An}$ & $2 / 3$ & 0 & 1
\end{tabular}

The addition of this step is justified by the observation of $\mathrm{Al}_{2} \mathrm{O}_{3}$-enrichment of pyroxenes and breakdown of early formed anorthites in $\mathrm{SiO}_{2}$-poor rocks (Carmichael et al 1970).

Equations used to construct the composition matrices are given in table 3 . Also are given the step numbers where the formulas derived from these equations have been used in the following section.

\section{Calculation steps}

The oxides and their molecular weights employed in the calculation of the norm are given in table 2 . The normative minerals considered, and their formulas and symbols are also given. While Kelsey (1965) used $3 \mathrm{CaO} \cdot \mathrm{P}_{2} \mathrm{O}_{5} \cdot 1 / 3 \mathrm{CaF}$ for apatite, Verma et al $(2002,2003)$ have used $3 \mathrm{CaO} \cdot \mathrm{P}_{2} \mathrm{O}_{5} \cdot 1 / 3 \mathrm{CaF}_{2}$ which is the correct formula. Verma et al (2002, 2003 ) also used a formula $3 \mathrm{CaO} \cdot \mathrm{P}_{2} \mathrm{O}_{5} \cdot 1 / 3 \mathrm{CaO}$ in the absence of analytical data on $\mathrm{F}$ which is most often the case. The latter, however, is not necessary and the standard formula should be used instead. In the present study the formula of $9 \mathrm{CaO} \cdot 3 \mathrm{P}_{2} \mathrm{O}_{5}$. $\mathrm{CaF}_{2}$ has been used and the discrepancy arising due to the absence of data on $\mathrm{F}$ is taken care of in the final sum of the normative minerals. Let us consider a rock of the following composition. The result of calculation at each step is given in a box.

\begin{tabular}{lr}
\hline Oxides & Wt.\% \\
\hline $\mathrm{SiO}_{2}$ & 28.03 \\
$\mathrm{TiO}_{2}$ & 0.97 \\
$\mathrm{Al}_{2} \mathrm{O}_{3}$ & 7.84 \\
$\mathrm{FeO}$ & 57.27 \\
$\mathrm{MgO}$ & 0.50 \\
$\mathrm{CaO}$ & 4.27 \\
$\mathrm{~K}_{2} \mathrm{O}$ & 0.45 \\
$\mathrm{P}_{2} \mathrm{O}_{5}$ & 0.39 \\
Total & 99.72 \\
\hline
\end{tabular}


Table 3. Equations used to construct the composition matrices and the step numbers at which the formulas derived from their inverse matrices have been used.

\begin{tabular}{|c|c|}
\hline Steps & Equations \\
\hline Step 3 & $\begin{array}{l}\mathrm{An}=0 \mathrm{C}+1 \mathrm{An} \\
\mathrm{Wo}=-1 \mathrm{C}+1 \mathrm{An}\end{array}$ \\
\hline Step 4 & $\begin{array}{l}\mathrm{Wo}=-1 \mathrm{C}+1 \mathrm{An}+0 \mathrm{Ab}+0 \mathrm{Hm} \\
\mathrm{Ab}=0 \mathrm{C}+0 \mathrm{An}+1 \mathrm{Ab}+0 \mathrm{Hm} \\
\mathrm{Hm}=0 \mathrm{C}+0 \mathrm{An}+0 \mathrm{Ab}+1 \mathrm{Hm} \\
\mathrm{Ac}=-1 \mathrm{C}+0 \mathrm{An}+1 \mathrm{Ab}+1 \mathrm{Hm}\end{array}$ \\
\hline Step 6 & $\begin{array}{l}\mathrm{Ab}=0 \mathrm{C}+1 \mathrm{Ab} \\
\mathrm{Ns}=-1 \mathrm{C}+1 \mathrm{Ab}\end{array}$ \\
\hline Step 7 & $\begin{array}{l}\mathrm{Ns}=-1 \mathrm{C}+1 \mathrm{Ab}+0 \mathrm{Or} \\
\mathrm{Or}=0 \mathrm{C}+0 \mathrm{Ab}+1 \mathrm{Or} \\
\mathrm{Ks}=-1 \mathrm{C}+0 \mathrm{Ab}+1 \mathrm{Or}\end{array}$ \\
\hline $\begin{array}{l}\text { Steps } 11 \\
\text { and } 13\end{array}$ & $\begin{array}{l}\mathrm{Ol}=-1 \mathrm{Q}+2 \mathrm{Hy} \\
\mathrm{Hy}=0 \mathrm{Q}+1 \mathrm{Hy}\end{array}$ \\
\hline Step 14 & $\begin{array}{l}\mathrm{Ol}=-1 \mathrm{Q}+2 \mathrm{Hy}+0 \mathrm{Ab} \\
\mathrm{Ne}=-4 \mathrm{Q}+0 \mathrm{Hy}+1 \mathrm{Ab} \\
\mathrm{Ab}=0 \mathrm{Q}+0 \mathrm{Hy}+1 \mathrm{Ab}\end{array}$ \\
\hline Step 15 & $\begin{array}{l}\mathrm{Ol}=-1 \mathrm{Q}+2 \mathrm{Hy}+0 \mathrm{Ab}+0 \mathrm{Or} \\
\mathrm{Ne}=-4 \mathrm{Q}+0 \mathrm{Hy}+1 \mathrm{Ab}+0 \mathrm{Or} \\
\mathrm{Lc}=-2 \mathrm{Q}+0 \mathrm{Hy}+0 \mathrm{Ab}+1 \mathrm{Or} \\
\mathrm{Or}=0 \mathrm{Q}+0 \mathrm{Hy}+0 \mathrm{Ab}+1 \mathrm{Or}\end{array}$ \\
\hline Step 16 & $\begin{array}{l}\mathrm{Ol}=-1 \mathrm{Q}+2 \mathrm{Hy}+0 \mathrm{Ab}+0 \mathrm{Or}+0 \mathrm{Wo} \\
\mathrm{Ne}=-4 \mathrm{Q}+0 \mathrm{Hy}+1 \mathrm{Ab}+0 \mathrm{Or}+0 \mathrm{Wo} \\
\mathrm{Lc}=-2 \mathrm{Q}+0 \mathrm{Hy}+0 \mathrm{Ab}+1 \mathrm{Or}+0 \mathrm{Wo} \\
\mathrm{Cs}=-1 \mathrm{Q}+0 \mathrm{Hy}+0 \mathrm{Ab}+0 \mathrm{Or}+2 \mathrm{Wo} \\
\mathrm{Wo}=0 \mathrm{Q}+0 \mathrm{Hy}+0 \mathrm{Ab}+0 \mathrm{Or}+1 \mathrm{Wo}\end{array}$ \\
\hline Step 17 & $\begin{array}{l}\mathrm{Ol}=-1 \mathrm{Q}+2 \mathrm{Hy}+0 \mathrm{Ab}+0 \mathrm{Or}+0 \mathrm{Di} \\
\mathrm{Ne}=-4 \mathrm{Q}+0 \mathrm{Hy}+1 \mathrm{Ab}+0 \mathrm{Or}+0 \mathrm{Di} \\
\mathrm{Lc}=-2 \mathrm{Q}+0 \mathrm{Hy}+0 \mathrm{Ab}+1 \mathrm{Or}+0 \mathrm{Di} \\
\mathrm{Cs}=-1 \mathrm{Q}-2 \mathrm{Hy}+0 \mathrm{Ab}+0 \mathrm{Or}+2 \mathrm{Di} \\
\mathrm{Di}=0 \mathrm{Q}+0 \mathrm{Hy}+0 \mathrm{Ab}+0 \mathrm{Or}+1 \mathrm{Di}\end{array}$ \\
\hline Step 18 & $\begin{array}{l}\mathrm{Ol}=-1 \mathrm{Q}+2 \mathrm{Hy}+0 \mathrm{Ab}+0 \mathrm{Or}+0 \mathrm{Di} \\
\mathrm{Ne}=-4 \mathrm{Q}+0 \mathrm{Hy}+1 \mathrm{Ab}+0 \mathrm{Or}+0 \mathrm{Di} \\
\mathrm{Lc}=-2 \mathrm{Q}+0 \mathrm{Hy}+0 \mathrm{Ab}+1 \mathrm{Or}+0 \mathrm{Di} \\
\mathrm{Cs}=-1 \mathrm{Q}-2 \mathrm{Hy}+0 \mathrm{Ab}+0 \mathrm{Or}+2 \mathrm{Di} \\
\mathrm{Kp}=-4 \mathrm{Q}+0 \mathrm{Hy}+0 \mathrm{Ab}+1 \mathrm{Or}+0 \mathrm{Di}\end{array}$ \\
\hline Step 20 & $\begin{array}{l}\mathrm{Cs}=0 \mathrm{Q}+1 \mathrm{Cs}+0 \mathrm{An} \\
\mathrm{C}=-\frac{3}{2} \mathrm{Q}-\frac{1}{2} \mathrm{Cs}+1 \mathrm{An} \\
\mathrm{An}=0 \mathrm{Q}+0 \mathrm{Cs}+1 \mathrm{An}\end{array}$ \\
\hline
\end{tabular}

1. Convert the weight percents of the oxides to mole numbers by dividing by the respective molecular weights.

Add moles $\mathrm{MnO}$ and $\mathrm{NiO}$ to moles $\mathrm{FeO}$. Add moles $\mathrm{BaO}$ and $\mathrm{SrO}$ to moles $\mathrm{CaO}$.

\begin{tabular}{ll}
\hline Oxides & Moles \\
\hline $\mathrm{SiO}_{2}$ & 0.466511 \\
$\mathrm{TiO}_{2}$ & 0.012145 \\
$\mathrm{Al}_{2} \mathrm{O}_{3}$ & 0.076892 \\
$\mathrm{FeO}$ & 0.797139 \\
$\mathrm{MgO}$ & 0.012406 \\
$\mathrm{CaO}$ & 0.076145 \\
$\mathrm{~K}_{2} \mathrm{O}$ & 0.004777 \\
$\mathrm{P}_{2} \mathrm{O}_{5}$ & 0.002748 \\
\hline
\end{tabular}

Calculate the minerals Z, Py, Cm, Ap, Cc and $\mathrm{Il}$ as given below and adjust the amounts of $\mathrm{SiO}_{2}, \mathrm{FeO}$ and $\mathrm{CaO}$ to be used for further calculations.

$$
\begin{gathered}
\mathrm{Z}_{1}=\mathrm{ZrO}_{2}, \quad \mathrm{SiO}_{2}=\mathrm{SiO}_{2}-\mathrm{Z}_{1}, \\
\mathrm{Py}_{1}=\frac{\mathrm{S}}{2}, \quad \mathrm{Cm}_{1}=\mathrm{Cr}_{2} \mathrm{O}_{3},
\end{gathered}
$$$$
\mathrm{FeO}=\mathrm{FeO}-\mathrm{Py}_{1}-\mathrm{Cm}_{1}, \quad \mathrm{Ap}_{1}=\frac{\mathrm{P}_{2} \mathrm{O}_{5}}{3},
$$

$$
\begin{aligned}
\mathrm{Cc}_{1} & =\mathrm{CO}_{2}, \quad \mathrm{Fr}_{1}=\frac{\mathrm{F}}{2}, \\
\mathrm{CaO} & =\mathrm{CaO}-10 \mathrm{Ap}_{1}-\mathrm{Cc}_{1}-\mathrm{Fr}_{1} \\
\mathrm{Il}_{1} & =\mathrm{TiO}_{2}
\end{aligned}
$$$$
\begin{aligned}
& \mathrm{Z}_{1}=0, \mathrm{SiO}_{2}=0.466511 \\
& \mathrm{Py}_{1}=0, \mathrm{Cm}_{1}=0, \mathrm{FeO}=0.797139 \\
& \mathrm{Ap}_{1}=0.000916, \mathrm{Cc}_{1}=0, \mathrm{Fr}_{1}=0 \\
& \mathrm{CaO}=0.066985 \\
& \mathrm{Il}_{1}=0.012145
\end{aligned}
$$

2. Calculate the provisional $\mathrm{SiO}_{2}$ and $\mathrm{Al}_{2} \mathrm{O}_{3}$ saturated minerals as follows:

$$
\begin{aligned}
& \mathrm{Or}_{\mathrm{p}}=\mathrm{K}_{2} \mathrm{O}, \quad \mathrm{Ab}_{\mathrm{p}}=\mathrm{Na}_{2} \mathrm{O}, \quad \mathrm{An}_{\mathrm{p}}=\mathrm{CaO}, \\
& \mathrm{En}_{\mathrm{p}}=\mathrm{MgO}, \quad \mathrm{Fs}_{\mathrm{p}}=\mathrm{FeO}, \quad \mathrm{Hm}_{\mathrm{p}}=\mathrm{Fe}_{2} \mathrm{O}_{3}, \\
& \mathrm{C}_{\mathrm{p}}=\mathrm{Al}_{2} \mathrm{O}_{3}-\mathrm{CaO}-\mathrm{Na}_{2} \mathrm{O}-\mathrm{K}_{2} \mathrm{O}
\end{aligned}
$$$$
\begin{aligned}
& \mathrm{Or}_{\mathrm{p}}=0.004777, \mathrm{Ab}_{\mathrm{p}}=0, \mathrm{An}_{\mathrm{p}}=0.066986 \\
& \mathrm{En}_{\mathrm{p}}=0.012406, \mathrm{Fs}_{\mathrm{p}}=0.797139, \mathrm{Hm}_{\mathrm{p}}=0 \\
& \mathrm{C}_{\mathrm{p}}=0.005128
\end{aligned}
$$ 
3. If $\mathrm{C}_{\mathrm{p}}<0$ :

$$
\begin{aligned}
& \mathrm{An}_{1}=\mathrm{C}_{\mathrm{p}}+\mathrm{An}_{\mathrm{p}} \quad \text { else } \quad \mathrm{An}_{1}=\mathrm{An}_{\mathrm{p}} \\
& \mathrm{Wo}_{1}=-\mathrm{C}_{\mathrm{p}} \quad \text { else } \quad \mathrm{Wo}_{1}=0
\end{aligned}
$$$$
\begin{aligned}
& \mathrm{An}_{1}=0.066986 \\
& \mathrm{Wo}_{1}=0
\end{aligned}
$$

4. If $\mathrm{An}_{1}<0$ :

$$
\begin{aligned}
& \mathrm{Wo}_{2}=0 \\
& \mathrm{Ab}_{1}=0 \\
& \mathrm{Hm}_{1}=0 \\
& \mathrm{Ac}_{1}=0
\end{aligned}
$$$$
\mathrm{Wo}_{2}=\mathrm{An}_{\mathrm{p}} \quad \text { else } \quad \mathrm{Wo}_{2}=\mathrm{Wo}_{1}
$$$$
A b_{1}=C_{p}+A n_{p}+A b_{p} \quad \text { else } \quad A b_{1}=A b_{p}
$$$$
\mathrm{Hm}_{1}=\mathrm{C}_{\mathrm{p}}+\mathrm{An}_{\mathrm{p}}+\mathrm{Hm}_{\mathrm{p}} \text { else } \mathrm{Hm}_{1}=\mathrm{Hm}_{\mathrm{p}}
$$$$
\mathrm{Ac}_{1}=-\mathrm{C}_{\mathrm{p}}-\mathrm{An}_{\mathrm{p}} \quad \text { else } \quad \mathrm{Ac}_{1}=0
$$

5. If $\mathrm{Hm}_{1}<0$ :

$$
\begin{aligned}
& \mathrm{Ac}_{2}=\mathrm{Hm}_{\mathrm{p}} \quad \text { else } \mathrm{Ac}_{2}=\mathrm{Ac}_{1} \\
& \mathrm{Hm}_{2}=\mathrm{Hm}_{\mathrm{p}}-\mathrm{Ac}_{2} \\
& \mathrm{Ab}_{2}=\mathrm{Ab}_{\mathrm{p}}-\mathrm{Ac}_{2}
\end{aligned}
$$$$
\text { if } \mathrm{An}_{1}<0, \quad \mathrm{C}_{1}=\mathrm{An}_{1}+\mathrm{Ac}_{2} \quad \text { else } \quad \mathrm{C}_{1}=0
$$

$$
\begin{aligned}
& \mathrm{Ac}_{2}=0 \\
& \mathrm{Hm}_{2}=0 \\
& \mathrm{Ab}_{2}=0 \\
& \mathrm{C}_{1}=0
\end{aligned}
$$

6. If $\mathrm{C}_{1}<0$ :

$$
\begin{aligned}
& \mathrm{Ab}_{3}=\mathrm{C}_{1}+\mathrm{Ab}_{2} \quad \text { else } \quad \mathrm{Ab}_{3}=\mathrm{Ab}_{2} \\
& \mathrm{Ns}_{1}=-\mathrm{C}_{1} \quad \text { else } \quad \mathrm{Ns}_{1}=0
\end{aligned}
$$

$$
\begin{aligned}
& \mathrm{Ab}_{3}=0 \\
& \mathrm{Ns}_{1}=0
\end{aligned}
$$

7. If $A b_{3}<0$ :

$$
\begin{aligned}
& \mathrm{Ns}_{2}=\mathrm{Ab}_{2} \quad \text { else } \quad \mathrm{Ns}_{2}=\mathrm{Ns}_{1} \\
& \mathrm{Or}_{1}=\mathrm{C}_{1}+\mathrm{Ab}_{2}+\mathrm{Or}_{\mathrm{p}} \quad \text { else } \mathrm{Or}_{1}=\mathrm{Or}_{\mathrm{p}} \\
& \mathrm{Ks}_{1}=-\mathrm{C}_{1}-\mathrm{Ab}_{2} \quad \text { else } \quad \mathrm{Ks}_{1}=0
\end{aligned}
$$

$$
\begin{aligned}
& \mathrm{Ns}_{2}=0 \\
& \mathrm{Or}_{1}=0.004777 \\
& \mathrm{Ks}_{1}=0
\end{aligned}
$$

8. If $\mathrm{Il}_{1}>\mathrm{Fs}_{\mathrm{p}}$ :

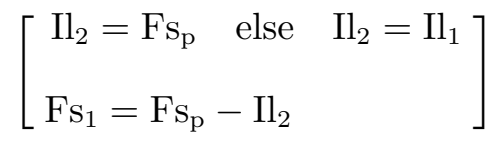

if $\mathrm{Il}_{1}>\mathrm{Il}_{2}, \quad \operatorname{Tn}_{1}=\mathrm{Il}_{1}-\mathrm{Il}_{2} \quad$ else $\quad \operatorname{Tn}_{1}=0$

if $\operatorname{Tn}_{1}>\mathrm{Wo}_{2}, \quad \mathrm{Tn}_{2}=\mathrm{Wo}_{2} \quad$ else $\quad \operatorname{Tn}_{2}=\mathrm{Tn}_{1}$

if $\operatorname{Tn}_{2}<0, \quad \operatorname{Tn}_{3}=0 \quad$ else $\quad \operatorname{Tn}_{3}=\operatorname{Tn}_{2}$

$\mathrm{Ru}_{1}=\mathrm{Tn}_{1}-\mathrm{Tn}_{3}$

$\mathrm{Wo}_{3}=\mathrm{Wo}_{2}-\mathrm{Tn}_{3}$

if $\mathrm{Hm}_{2}<\mathrm{Fs}_{1}, \quad \mathrm{Mt}_{1}=\mathrm{Hm}_{2} \quad$ else $\quad \mathrm{Mt}_{1}=\mathrm{Fs}_{1}$

$\mathrm{Fs}_{2}=\mathrm{Fs}_{1}-\mathrm{Mt}_{1}$

if $\left(\mathrm{En}_{\mathrm{p}}+\mathrm{Fs}_{2}\right)>\mathrm{Wo}_{3}$,

$\mathrm{Hy}_{1}=\left(\mathrm{En}_{\mathrm{p}}+\mathrm{Fs}_{2}\right)-\mathrm{Wo}_{3}$ else $\mathrm{Hy}_{1}=0$

if $\mathrm{Wo}_{3}>\left(\mathrm{En}_{\mathrm{p}}+\mathrm{Fs}_{2}\right), \quad \mathrm{Di}_{1}=\mathrm{En}_{\mathrm{p}}+\mathrm{Fs}_{2}$

else $\mathrm{Di}_{1}=\mathrm{Wo}_{3}$

$\mathrm{Wo}_{4}=\mathrm{Wo}_{3}-\mathrm{Di}_{1}$ 


\begin{tabular}{|l|}
\hline $\mathrm{Il}_{2}=0.012145$ \\
$\mathrm{Fs}_{1}=0.784994$ \\
$\mathrm{Tn}_{1}=0$ \\
$\mathrm{Tn}_{2}=0$ \\
$\mathrm{Tn}_{3}=0$ \\
$\mathrm{Ru}_{1}=0$ \\
$\mathrm{Wo}_{3}=0$ \\
$\mathrm{Mt}_{1}=0$ \\
$\mathrm{Fs}_{2}=0.784994$ \\
$\mathrm{Hy}_{1}=0.7974$ \\
$\mathrm{Di}_{1}=0$ \\
$\mathrm{Wo}_{4}=0$
\end{tabular}

9. Reset the provisional amount of the following minerals as follows:

$$
\begin{aligned}
& \mathrm{Or}_{\mathrm{p}}=\mathrm{Or}_{1}, \mathrm{Ab}_{\mathrm{p}}=\mathrm{Ab}_{3}, \quad \mathrm{An}_{\mathrm{p}}=\mathrm{An}_{1}, \\
& \mathrm{Di}_{\mathrm{p}}=\mathrm{Di}_{1}, \quad \mathrm{Hy}_{\mathrm{p}}=\mathrm{Hy}_{1} \\
& \mathrm{Ac}_{\mathrm{p}}=\mathrm{Ac}_{2}, \quad \mathrm{Ks}_{\mathrm{p}}=\mathrm{Ks}_{1}, \quad \mathrm{Ns}_{\mathrm{p}}=\mathrm{Ns}_{2}, \\
& \mathrm{Wo}_{\mathrm{p}}=\mathrm{Wo}_{4}, \quad \mathrm{Tn}_{\mathrm{p}}=\mathrm{Tn}_{3}
\end{aligned}
$$$$
\begin{aligned}
& \mathrm{Or}_{\mathrm{p}}=0.004777, \mathrm{Ab}_{\mathrm{p}}=0 \\
& \mathrm{An}_{\mathrm{p}}=0.066986, \\
& \mathrm{Di}_{\mathrm{p}}=0, \mathrm{Hy}_{\mathrm{p}}=0.7974 \\
& \mathrm{Ac}_{\mathrm{p}}=0, \mathrm{Ks}_{\mathrm{p}}=0, \mathrm{Ns}_{\mathrm{p}}=0 \\
& \mathrm{Wo}_{\mathrm{p}}=0, \mathrm{Tn}_{\mathrm{p}}=0
\end{aligned}
$$

10. Calculate provisional quartz as:

$$
\begin{aligned}
& \mathrm{Q}_{1}= \mathrm{SiO}_{2}-\left(6 \mathrm{Or}_{\mathrm{p}}+6 \mathrm{Ab}_{\mathrm{p}}+2 \mathrm{An}_{\mathrm{p}}+2 \mathrm{Di}_{\mathrm{p}}\right. \\
&\left.+\mathrm{Hy}_{\mathrm{p}}+4 \mathrm{Ac}_{\mathrm{p}}+\mathrm{Ks}_{\mathrm{p}}+\mathrm{Ns}_{\mathrm{p}}+\mathrm{Wo}_{\mathrm{p}}+\mathrm{Tn}_{\mathrm{p}}\right) \\
& \mathrm{Q}_{1}=-0.493525
\end{aligned}
$$

11. If $\mathrm{Q}_{1}<0$ :

$$
\mathrm{Ol}_{1}=-\mathrm{Q}_{1} \quad \text { else } \quad \mathrm{Ol}_{1}=0
$$

$$
\begin{aligned}
\mathrm{Hy}_{2} & =2 \mathrm{Q}_{1}+\mathrm{Hy}_{\mathrm{p}} \\
\mathrm{Ol}_{1} & =0.493525 \\
\mathrm{Hy}_{2} & =-0.189649
\end{aligned}
$$

12. If $\mathrm{Hy}_{2}<0$ :

$$
\begin{aligned}
& \mathrm{Pf}_{1}=\mathrm{Tn}_{\mathrm{p}}+\mathrm{Hy}_{2} \quad \text { else } \mathrm{Pf}_{1}=0 \\
& \text { if } \mathrm{Pf}_{1}<0, \quad \mathrm{Pf}_{2}=\mathrm{Tn}_{\mathrm{p}} \text { else } \mathrm{Pf}_{2}=\mathrm{Pf}_{1} \\
& \mathrm{Tn}_{4}=\mathrm{Tn}_{\mathrm{p}}-\mathrm{Pf}_{2} \\
& \mathrm{Q}_{2}=\mathrm{Q}_{1}+\mathrm{Pf}_{2}
\end{aligned}
$$

$$
\begin{aligned}
& \mathrm{Pf}_{1}=-0.189649 \\
& \mathrm{Pf}_{2}=0 \\
& \mathrm{Tn}_{4}=0 \\
& \mathrm{Q}_{2}=-0.493525
\end{aligned}
$$

13. If $\mathrm{Q}_{\mathrm{p}}<0$ :

$$
\begin{aligned}
& \mathrm{Ol}_{2}=-\mathrm{Q}_{2} \text { else } \mathrm{Ol}_{2}=0 \\
& \mathrm{Hy}_{3}=2 \mathrm{Q}_{2}+\mathrm{Hy}_{\mathrm{p}}
\end{aligned}
$$

$$
\begin{aligned}
& \mathrm{Ol}_{2}=0.493525 \\
& \mathrm{Hy}_{3}=-0.189649
\end{aligned}
$$

14. If $\mathrm{Hy}_{3}<0$ :

$$
\mathrm{Ol}_{3}=0.5 \mathrm{Hy}_{\mathrm{p}} \quad \text { else } \quad \mathrm{Ol}_{3}=\mathrm{Ol}_{2}
$$

$\mathrm{Ne}_{1}=-0.25 \mathrm{Q}_{2}-0.125 \mathrm{Hy}_{\mathrm{p}} \quad$ else $\quad \mathrm{Ne}_{1}=0$

$$
\begin{gathered}
\mathrm{Ab}_{4}=0.25 \mathrm{Q}_{2}+0.125 \mathrm{Hy}_{\mathrm{p}}+\mathrm{Ab}_{\mathrm{p}} \\
\text { else } \quad \mathrm{Ab}_{4}=\mathrm{Ab}_{\mathrm{p}}
\end{gathered}
$$

$$
\begin{aligned}
& \mathrm{Ol}_{3}=0.3987 \\
& \mathrm{Ne}_{1}=0.023706 \\
& \mathrm{Ab}_{4}=-0.023706
\end{aligned}
$$


15. If $\mathrm{Ab}_{4}<0$ :

$$
\begin{gathered}
\mathrm{Ne}_{2}=\mathrm{Ab}_{\mathrm{p}} \quad \text { else } \quad \mathrm{Ne}_{2}=\mathrm{Ne}_{1} \\
\mathrm{Lc}_{1}=-0.5 \mathrm{Q}_{2}-0.25 \mathrm{Hy}_{\mathrm{p}}-2 \mathrm{Ab}_{\mathrm{p}} \\
\text { else } \quad \mathrm{Lc}_{1}=0 \\
\mathrm{Or}_{2}=0.5 \mathrm{Q}_{2}+0.25 \mathrm{Hy}_{\mathrm{p}}+2 \mathrm{Ab}_{\mathrm{p}}+\mathrm{Or}_{\mathrm{p}} \\
\text { else } \mathrm{Or}_{2}=\mathrm{Or}_{\mathrm{p}}
\end{gathered}
$$

$$
\begin{aligned}
& \mathrm{Ne}_{2}=0 \\
& \mathrm{Lc}_{1}=0.474124 \\
& \mathrm{Or}_{2}=-0.042635
\end{aligned}
$$

16. If $\mathrm{Or}_{2}<0$ :

$$
\begin{aligned}
& \mathrm{Lc}_{2}=\mathrm{Or}_{\mathrm{p}} \quad \text { else } \quad \mathrm{Lc}_{2}=\mathrm{Lc}_{1} \\
& \mathrm{Cs}_{1}=-\mathrm{Q}_{2}-0.5 \mathrm{Hy}_{\mathrm{p}}-4 \mathrm{Ab}_{\mathrm{p}}-2 \mathrm{Or}_{\mathrm{p}} \\
& \text { else } \quad \mathrm{Cs}_{1}=0 \\
& \mathrm{Wo}_{5}=2 \mathrm{Q}_{2}+\mathrm{Hy}_{\mathrm{p}}+8 \mathrm{Ab}_{\mathrm{p}}+4 \mathrm{Or}_{\mathrm{p}}+\mathrm{Wo}_{\mathrm{p}} \\
& \text { else } \quad \mathrm{Wo}_{5}=\mathrm{Wo}_{\mathrm{p}}
\end{aligned}
$$

if $\mathrm{Wo}_{5}<0, \quad \mathrm{Cs}_{2}=0.5 \mathrm{Wo}_{\mathrm{p}} \quad$ else $\quad \mathrm{Cs}_{2}=0$

$$
\begin{aligned}
& \mathrm{Lc}_{2}=0.004777 \\
& \mathrm{Cs}_{1}=0.085270 \\
& \mathrm{Wo}_{5}=-0.17054 \\
& \mathrm{Cs}_{2}=0
\end{aligned}
$$

17. If $\mathrm{Wo}_{5}<0$ :

$$
\begin{aligned}
& \mathrm{Ol}_{4}=-0.5 \mathrm{Q}_{2}+0.25 \mathrm{Hy}_{\mathrm{p}}-2 \mathrm{Ab}_{\mathrm{p}}-\mathrm{Or}_{\mathrm{p}} \\
& \text { else } \quad \mathrm{Ol}_{4}=\mathrm{Ol}_{3} \\
& \mathrm{Cs}_{3}=\mathrm{Cs}_{2}-0.5 \mathrm{Q}_{2}-0.25 \mathrm{Hy}_{\mathrm{p}}-2 \mathrm{Ab}_{\mathrm{p}}-\mathrm{Or}_{\mathrm{p}} \\
& \text { else } \quad \mathrm{Cs}_{3}=\mathrm{Cs}_{1}
\end{aligned}
$$

$$
\begin{gathered}
\mathrm{Di}_{2}=\mathrm{Q}_{2}+0.5 \mathrm{Hy}_{\mathrm{p}}+4 \mathrm{Ab}_{\mathrm{p}}+2 \mathrm{Or}_{\mathrm{p}}+\mathrm{Di}_{\mathrm{p}} \\
\text { else } \quad \mathrm{Di}_{2}=\mathrm{Di}_{\mathrm{p}}
\end{gathered}
$$

$\mathrm{Ol}_{4}=0.441335$
$\mathrm{Cs}_{3}=0.042635$
$\mathrm{Di}_{2}=-0.08527$

18. If $\mathrm{Di}_{2}<0$ :

$$
\begin{aligned}
\mathrm{Ol}_{5}= & 0.5 \mathrm{Hy}_{\mathrm{p}}+0.5 \mathrm{Di}_{\mathrm{p}} \quad \text { else } \quad \mathrm{Ol}_{5}=\mathrm{Ol}_{4} \\
\mathrm{Lc}_{3}= & 0.5 \mathrm{Q}_{2}+0.25 \mathrm{Hy}_{\mathrm{p}}+2 \mathrm{Ab}_{\mathrm{p}}+2 \mathrm{Or}_{\mathrm{p}} \\
& +0.5 \mathrm{Di}_{\mathrm{p}} \quad \text { else } \quad \mathrm{Lc}_{3}=\mathrm{Lc}_{1} \\
\mathrm{Cs}_{4}= & 0.5 \mathrm{Di}_{\mathrm{p}}+\mathrm{Cs}_{2} \quad \text { else } \quad \mathrm{Cs}_{4}=\mathrm{Cs}_{3} \\
\mathrm{Kp}_{1}= & -0.5 \mathrm{Q}_{2}-0.25 \mathrm{Hy}_{\mathrm{p}}-2 \mathrm{Ab}_{\mathrm{p}}-\mathrm{Or}_{\mathrm{p}} \\
& -0.5 \mathrm{Di}_{\mathrm{p}} \quad \text { else } \quad \mathrm{Kp}_{1}=0 \\
\mathrm{Ol}_{5}= & 0.3987 \\
\mathrm{Lc}_{3}= & -0.03786 \\
\mathrm{Cs}_{4}= & 0 \\
\mathrm{Kp}_{1}= & 0.042635
\end{aligned}
$$

19. If $\mathrm{Lc}_{3}<0$ :

$$
\begin{aligned}
& \mathrm{Lc}_{4}=0 \quad \text { else } \mathrm{Lc}_{4}=\mathrm{Lc}_{2} \\
& \mathrm{Kp}_{2}=\mathrm{Lc}_{2} \text { else } \mathrm{Kp}_{2}=\mathrm{Kp}_{1} \\
& \text { if both }\left(\mathrm{Lc}_{3}>0\right) \text { and }\left(\mathrm{Kp}_{2}>0\right) \text {, } \\
& \qquad \mathrm{Lc}_{5}=\mathrm{Lc}_{3} \text { else } \mathrm{Lc}_{5}=\mathrm{Lc}_{4}
\end{aligned}
$$

$$
\begin{aligned}
& \mathrm{Lc}_{4}=0 \\
& \mathrm{Kp}_{2}=0.004777 \\
& \mathrm{Lc}_{5}=0
\end{aligned}
$$

20. This step is an extension to the classic CIPW norm calculation and takes care of the residual $\mathrm{SiO}_{2}$ deficiency, if any, existing even after the formation of Kp by the breakdown of Lc.

$$
\begin{aligned}
& \mathrm{Q}_{3}=2\left(\mathrm{Kp}_{2}-\mathrm{Kp}_{1}\right) \\
& \text { if } \mathrm{C}_{\mathrm{p}}<0, \quad \mathrm{C}_{2}=0 \text { else } \mathrm{C}_{2}=\mathrm{C}_{\mathrm{p}}
\end{aligned}
$$




$$
\begin{aligned}
& \mathrm{Q}_{3}=-0.075716 \\
& \mathrm{C}_{2}=0.005128 \\
& \mathrm{Cs}_{5}=0.025238 \\
& \mathrm{C}_{3}=0.050477 \\
& \mathrm{An}_{2}=0.016509
\end{aligned}
$$$$
\mathrm{Cs}_{5}=\mathrm{Cs}_{4}-\frac{1}{3} \mathrm{Q}_{3}
$$$$
\mathrm{C}_{3}=-\frac{2}{3} \mathrm{Q}_{3}
$$$$
\mathrm{An}_{2}=\mathrm{An}_{\mathrm{p}}+\frac{2}{3} \mathrm{Q}_{3}
$$

21. Assign molecular fractions to the normative minerals as follows:

$$
\begin{aligned}
& \text { if } \mathrm{Q}_{2}<0, \quad \mathrm{Q}=0 \text { else } \mathrm{Q}=\mathrm{Q}_{2} \\
& \text { if } \mathrm{Or}_{2}<0, \quad \mathrm{Or}=0 \text { else } \mathrm{Or}=\mathrm{Or}_{2} \\
& \text { if } \quad \mathrm{Ab}_{4}<0, \quad \mathrm{Ab}=0 \quad \text { else } \quad \mathrm{Ab}=\mathrm{Ab}_{4} \\
& \text { if } \quad \mathrm{Di}_{2}<0, \quad \mathrm{Di}=0 \quad \text { else } \quad \mathrm{Di}=\mathrm{Di}_{2} \\
& \text { if } \mathrm{Hy}_{3}<0, \quad \mathrm{Hy}=0 \text { else } \mathrm{Hy}=\mathrm{Hy}_{3} \\
& \text { if } \quad \mathrm{WO}_{5}<0, \quad \mathrm{Wo}=0 \quad \text { else } \quad \mathrm{Wo}=\mathrm{WO}_{5} \\
& \mathrm{C}=\mathrm{C}_{2}+\mathrm{C}_{3}, \quad \mathrm{An}=\mathrm{An}_{2}, \quad \mathrm{Ne}=\mathrm{Ne}_{2}, \\
& \mathrm{Ol}=\mathrm{Ol}_{5}, \quad \mathrm{Mt}=\mathrm{Mt}_{1} \\
& \mathrm{Il}=\mathrm{Il}_{2}, \quad \mathrm{Ap}=\mathrm{Ap}_{1}, \quad \mathrm{Ac}=\mathrm{Ac}_{\mathrm{p}}, \\
& \mathrm{Lc}=\mathrm{Lc}_{5}, \quad \mathrm{Ks}=\mathrm{Ks}_{1}, \quad \mathrm{Ns}=\mathrm{Ns}_{2} \\
& \mathrm{Cs}=\mathrm{Cs}_{5}, \quad \mathrm{Kp}=\mathrm{Kp}_{2}, \quad \mathrm{Hm}=\mathrm{Hm}_{2}, \\
& \mathrm{Tn}=\mathrm{Tn}_{4}, \quad \mathrm{Ru}=\mathrm{Ru}_{1} \\
& \mathrm{Pf}=\mathrm{Pf}_{2}, \quad \mathrm{Cc}=\mathrm{Cc}_{1}, \quad \mathrm{Py}=\mathrm{Py}_{1}, \\
& \mathrm{Cm}=\mathrm{Cm}_{1}, \quad \mathrm{Fr}=\mathrm{Fr}_{1}, \quad \mathrm{Z}=\mathrm{Z}_{1}
\end{aligned}
$$$$
\begin{array}{|l|}
\hline \mathrm{Q}=0 \\
\mathrm{Or}=0 \\
\mathrm{Ab}=0 \\
\mathrm{Di}=0 \\
\mathrm{Hy}=0
\end{array}
$$

$$
\begin{aligned}
& \mathrm{Wo}=0 \\
& \mathrm{C}=0.0556055, \mathrm{An}=0.016509 \\
& \mathrm{Ne}=0, \mathrm{Ol}=0.3987, \mathrm{Mt}=0 \\
& \mathrm{Il}=0.012145, \mathrm{Ap}=0.000916 \\
& \mathrm{Lc}=0, \mathrm{Ks}=0, \quad \mathrm{Ns}=0 \\
& \mathrm{Cs}=0.025238, \mathrm{Kp}=0.004777 \\
& \mathrm{Hm}=0, \mathrm{Tn}=0, \quad \mathrm{Ru}=0 \\
& \mathrm{Pf}=0, \mathrm{Cc}=0, \mathrm{Py}=0, \mathrm{Cm}=0, \\
& \mathrm{Fr}=0, \mathrm{Z}=0
\end{aligned}
$$

22. Calculate the formula weights of the normative minerals using the molecular weights of the oxides. For $\mathrm{FeO}$ and $\mathrm{CaO}$ use the molecular weights determined as follows:

$\mathrm{Mol}$ wt. $\mathrm{FeO}=\frac{\mathrm{Wt} . \%(\mathrm{FeO}+\mathrm{MnO}+\mathrm{NiO})}{\operatorname{Moles}(\mathrm{FeO}+\mathrm{MnO}+\mathrm{NiO})}$

Mol wt. $\mathrm{CaO}=\frac{\mathrm{Wt} . \%(\mathrm{CaO}+\mathrm{BaO}+\mathrm{SrO})}{\operatorname{Moles}(\mathrm{CaO}+\mathrm{BaO}+\mathrm{SrO})}$

Mol wt. $\mathrm{FeO}=71.8444$

Mol wt. $\mathrm{CaO}=56.0774$

23. Estimate the formula weights of the ferromagnesian minerals hypersthene, diopside and olivine as follows:

$$
\begin{aligned}
& \text { Fraction } \mathrm{Fe}=\frac{\mathrm{Fs}_{2}}{\left(\mathrm{En}_{\mathrm{p}}+\mathrm{Fs}_{2}\right)} \\
& \text { Fraction } \mathrm{Mg}=\frac{\mathrm{En}_{\mathrm{p}}}{\left(\mathrm{En}_{\mathrm{p}}+\mathrm{Fs}_{2}\right)}
\end{aligned}
$$

Formula wt. $\mathrm{Hy}=$ Fraction $\mathrm{Mg} \times \mathrm{Mol}$ wt. En + Fraction Fe $\times$ Mol wt. Fs

Formula wt. Di = Formula wt. Hy + Mol wt. Wo

Formula wt. $\mathrm{Ol}=$ Fraction $\mathrm{Mg} \times(\mathrm{Mol}$ wt. En + Mol wt. $\mathrm{MgO})+$ Fraction Fe $\times(\mathrm{Mol}$ wt. Fs $+\mathrm{Mol}$ wt. FeO $)$ 
\begin{tabular}{|l|}
\hline Fraction $\mathrm{Fe}=0.984442$ \\
Fraction $\mathrm{Mg}=0.015558$ \\
Formula wt. $\mathrm{Hy}=131.438$ \\
Formula wt. $\mathrm{Di}=247.5997$ \\
Formula wt. $\mathrm{Ol}=202.7917$
\end{tabular}

24. Determine the weight percentages of the normative minerals by multiplying their molecular fractions with respective formula weights.

$$
\begin{aligned}
& \mathrm{C}=5.669607, \quad \mathrm{An}=4.592971 \\
& \mathrm{Ol}=80.85302 \\
& \mathrm{Il}=1.842577, \quad \mathrm{Ap}=0.923732 \\
& \mathrm{Cs}=4.347064, \quad \mathrm{Kp}=1.511175
\end{aligned}
$$

25. Find the sum of the weight percentages of normative minerals. Add to it

$$
\begin{aligned}
& \text { Moles }(\mathrm{Py}+\mathrm{Fr}+\mathrm{Ap}) \times 15.9994 \\
& -(\text { Moles } \mathrm{Ap} \times 2+\text { Moles Fr }) \times 18.9984 \\
& + \text { Atoms } \mathrm{F} \times 18.9984+\text { Moles } \mathrm{Cs}_{2} \times 60.0843
\end{aligned}
$$

where $15.9994=$ At. wt. O, $18.9984=$ At. wt. F, $60.0843=$ Mol. wt. $\mathrm{SiO}_{2}$. This sum should exactly match with the sum of the weight percentages of the oxides. Sum of the weights of the normative minerals is 99.74015 . Because no pyrite or fluorite are in the norm; the first and second terms in the correction above become Moles Ap $\times 15.9994-$ Moles Ap $\times 2 \times 18.9984=$ $0.000916 \times 15.9994-0.000916 \times 2 \times 18.9984=$ -0.02015 . Because there was no fluorine in the input and $\mathrm{Cs}_{2}$ is zero as determined in step 16, the rest two terms amount to zero. Therefore, the corrected sum of the normative minerals will be $99.74015-0.02015=99.72000$.

$$
\begin{aligned}
& \text { Sum }(\text { oxides })=99.72 \\
& \text { Sum }(\text { normative minerals })=99.74015 \\
& \text { Corrected sum (normative minerals) }= \\
& 99.72
\end{aligned}
$$

\begin{tabular}{|c|c|c|}
\hline & \multicolumn{2}{|c|}{ Oxides (wt. \%) } \\
\hline & 1 & 2 \\
\hline $\mathrm{SiO}_{2}$ & 28.03 & 27.79 \\
\hline $\mathrm{TiO}_{2}$ & 0.97 & 0.96 \\
\hline $\mathrm{Al}_{2} \mathrm{O}_{3}$ & 7.84 & 7.77 \\
\hline $\mathrm{Fe}_{2} \mathrm{O}_{3}$ & 0.00 & 11.47 \\
\hline $\mathrm{FeO}$ & 57.27 & 46.45 \\
\hline $\mathrm{MgO}$ & 0.50 & 0.50 \\
\hline $\mathrm{CaO}$ & 4.27 & 4.23 \\
\hline $\mathrm{K}_{2} \mathrm{O}$ & 0.45 & 0.45 \\
\hline $\mathrm{P}_{2} \mathrm{O}_{5}$ & 0.39 & 0.39 \\
\hline \multirow[t]{3}{*}{ Total } & 99.72 & 100.00 \\
\hline & \multicolumn{2}{|c|}{ Norms (wt. \%) } \\
\hline & 1 & 2 \\
\hline $\mathrm{C}$ & 5.66961 & 0.51834 \\
\hline Or & 0.00000 & 2.63616 \\
\hline An & 4.59297 & 18.47367 \\
\hline Hy & 0.00000 & 3.60070 \\
\hline $\mathrm{Ol}$ & 80.85302 & 55.42251 \\
\hline Mt & 0.00000 & 16.62639 \\
\hline $\mathrm{Il}$ & 1.84258 & 1.82652 \\
\hline Ap & 0.92373 & 0.91568 \\
\hline Cs & 4.34706 & 0.00000 \\
\hline $\mathrm{Kp}$ & 1.51118 & 0.00000 \\
\hline Total & 99.74015 & 100.01997 \\
\hline $\begin{array}{l}\text { Corrected } \\
\text { total }\end{array}$ & 99.72000 & 100.00000 \\
\hline
\end{tabular}

The result is summarized below. Note that the effect of partitioning the total Fe between $\mathrm{Fe}^{2+}$ and $\mathrm{Fe}^{3+}$ is given in the columns numbered 2. The equation of Le Maitre (1976) has been used as the composition falls beyond the limits of the TAS diagram.

\section{Causes of mass discrepancy}

In the calculation of the CIPW norm, we start with definite amounts of different oxides and get at the end a set of minerals of definite quantities that can be formed by consuming these oxides. It is but natural that the weights of the oxides and that of the normative minerals should be the same, but it rarely happens so. Norms calculated from the same set of oxides in same quantities using different approaches do not always have identical values. Assuming all methods correctly estimate the norm, this kind of discrepancy can arise from the fact that different people use slightly different values for the oxide molecular weights. Sticking to a standard set of molecular weights would be preferable for achieving a standard norm. 
The difference between the totals of the oxides and that of the minerals may arise due to a lack of internal consistency in the implemented steps of calculation. The calculation procedure may not be working as expected in different composition ranges. A logically simple and easy to understand formulation of the calculation procedure is therefore desirable.

Once all logical and computational errors are dealt with, mass discrepancy can arise due to three causes. Firstly, corrected molecular weights of $\mathrm{FeO}$ and $\mathrm{CaO}$ need to be used because of the fact that moles of $\mathrm{MnO}$ and $\mathrm{NiO}$ are added to $\mathrm{FeO}$, and moles of $\mathrm{BaO}$ and $\mathrm{SrO}$ are added to $\mathrm{CaO}$. Corrected molecular weight of $\mathrm{FeO}$ can be easily estimated by dividing the sum of weights of $\mathrm{FeO}, \mathrm{MnO}$ and $\mathrm{NiO}$ by the sum of the molecular fractions of the three. Corrected molecular weight of $\mathrm{CaO}$ can be similarly estimated. The second cause of discrepancy is because the mineral formulas of pyrite $\left(\mathrm{FeS}_{2}\right)$, fluorite $\left(\mathrm{CaF}_{2}\right)$ and apatite $\left(9 \mathrm{CaO} \cdot 3 \mathrm{P}_{2} \mathrm{O}_{5}\right.$. $\left.\mathrm{CaF}_{2}\right)$ are defined partially or completely by chemical species other than oxides, whereas all chemical constituents are input in the form of oxides. The discrepancy, for example, in the case of pyrite can be removed if we calculate the formula weight of pyrite as mol.wt. $(\mathrm{FeO})+$ at.wt. $(\mathrm{S}) \times 2$ instead of as at.wt. $(\mathrm{Fe})+$ at.wt. $(\mathrm{S}) \times 2$. Appropriate corrections in the sum of the mineral weights should be applied to take care of this discrepancy if true formula weights are used. The third source of mass discrepancy is from significant rounding off errors. The only scope of introducing significant rounding off error in the procedure delineated above is in the 20th step. Care must be taken to avoid using $0.333333 \ldots$ in place of $1 / 3$ and $0.666666 \ldots$ in place of $2 / 3$ which can result in considerable mismatch between the sums in some cases.

If all $W_{0}$ is converted to $C_{s}$ in step 16, an equivalent amount of $\mathrm{SiO}_{2}$ must be added to the final sum.

Finally, it is to be noted that this budgeting exercise is just unnecessary as it has no effect on the calculated norms. However, it works as a beautiful check against inconsistencies in the input values and provides a guard against unsuspected errors.

\section{Discussion}

Although trace element and isotope geochemistry have surpassed the CIPW norm in the interpretation of igneous rocks, the role of CIPW norm remains unbeaten in the use of it in the classification of igneous rocks, that of the volcanic rocks in particular. Very precise discriminative parameters used in the classification of the igneous rocks demand for very precise calculation of the CIPW norm. There are a number of superior computer packages available for petrological purpose (PetroGraph, GeoPlot and SINCLAS, to name a few), but none of them reproduce the total of the oxides to the degree required. SINCLAS (Verma et al 2002), for example, even after using simplified calculation method of Kelsey (1965) and using variable atomic weights, has an inaccuracy of up to $0.002 \% \mathrm{~m} / \mathrm{m}$ (wt.\%). The new set of rules presented here use easy to understand linear algebraic equations and so, minimize the chance of error in the estimation of the normative minerals. Further, the steps for calculation of the norm are given in a programmer-friendly manner removing the scope of any ambiguity. Possible error due to the presence of $\mathrm{SiO}_{2}$ deficiency beyond the scope of the classic scheme of calculation has been preempted by introducing additional steps for calculation and steps to prevent erroneous estimation of the sum of the normative minerals have been provided. The method has been checked for about 11,200 analyses spanning a wide variety of rocks. A comparison with the results obtained from SINCLAS shows that there is insignificant difference between the calculated norms. However, with the present calculation scheme all factors contributing towards differences between the total of the analyses and the total of the norms have been completely resolved. For example, the norms for an ultrabasic rock analysis have been compared below with the results obtained from SINCLAS. When rounded to the third decimal place, norms compare well except for apatite. This difference is because of the fact that the formula for apatite in the present scheme is uniformly taken as $9 \mathrm{CaO} \cdot 3 \mathrm{P}_{2} \mathrm{O}_{5} \cdot \mathrm{CaF}_{2}$ (equivalent to $3 \mathrm{CaO} \cdot \mathrm{P}_{2} \mathrm{O}_{5} \cdot 1 / 3 \mathrm{CaF}_{2}$ ) whether or not $\mathrm{F}$ is there in the input and the final sum of the normative minerals are corrected accordingly. The

\begin{tabular}{lrlrr}
\hline & & & \multicolumn{2}{c}{ Norm (wt.\%) } \\
\cline { 3 - 5 } & $\begin{array}{r}\text { Oxide } \\
\text { (wt\%) }\end{array}$ & & $\begin{array}{c}\text { Present } \\
\text { study }\end{array}$ & SINCLAS \\
\hline $\mathrm{SiO}_{2}$ & 44.906 & Or & 1.81426 & 1.814 \\
$\mathrm{TiO}_{2}$ & 2.709 & $\mathrm{Alb}$ & 15.56949 & 15.569 \\
$\mathrm{Al}_{2} \mathrm{O}_{3}$ & 14.945 & $\mathrm{An}$ & 31.61230 & 31.612 \\
$\mathrm{Fe}_{2} \mathrm{O}_{3}$ & 1.927 & $\mathrm{Di}$ & 16.47141 & 16.471 \\
$\mathrm{FeO}$ & 12.850 & $\mathrm{Hy}$ & 7.62915 & 7.629 \\
$\mathrm{MnO}$ & 0.215 & Ol & 18.13576 & 18.135 \\
$\mathrm{MgO}$ & 9.057 & $\mathrm{Mt}$ & 2.79378 & 2.794 \\
$\mathrm{CaO}$ & 10.887 & $\mathrm{Il}$ & 5.14541 & 5.145 \\
$\mathrm{Na} \mathrm{N}_{2} \mathrm{O}$ & 1.840 & Ap & 0.83197 & 0.829 \\
$\mathrm{~K}_{2} \mathrm{O}$ & 0.307 & & & \\
$\mathrm{P}_{2} \mathrm{O}_{5}$ & 0.358 & & & \\
$\mathrm{Total}$ & 100.001 & Total & 100.00352 & 99.998 \\
& \multicolumn{5}{c}{ Corrected } & 100.00100 & \\
& \multicolumn{5}{c}{ total } \\
\hline
\end{tabular}


uncorrected sum of the normative minerals according to the proposed scheme is closer to the sum of the oxides with a difference of +0.00252 whereas SINCLAS has a difference of -0.00300 . The corrected total of the normative minerals as suggested in step 25 and explained in the previous section completely takes care of this difference.

The method described here keeps clear of the issue of the standardization of the oxidation ratio $\left(\mathrm{Fe}_{2} \mathrm{O}_{3} / \mathrm{FeO}\right)$ of igneous rocks. The user should use his discretion to choose a scheme preferable to him. It is also left to the user as to whether the input analysis should be recast to $100 \%$ total or not. These decisions are crucial as they potentially alter the final norms obtained. For example, norms obtained from analysis recast to a total of $100 \%$ will be different from the final norm that has been recast to $100 \%$ total, obtained without normalizing the input analysis. For the sake of oxidation ratios, Verma et al (2002, 2003) suggest three options, but favour the method of Middlemost (1989). Whenever possible, actual analyses of $\mathrm{Fe}_{2} \mathrm{O}_{3}$ and $\mathrm{FeO}$ should be used to fix this ratio (Le Bas et al 1986), but keeping in mind the possibility of post-emplacement oxidation. Alternatively, the equation of Le Maitre (1976),

$$
\begin{aligned}
\frac{\mathrm{FeO}}{\mathrm{FeO}+\mathrm{Fe}_{2} \mathrm{O}_{3}}= & 0.93-0.0042 \mathrm{SiO}_{2} \\
& -0.022\left(\mathrm{Na}_{2} \mathrm{O}+\mathrm{K}_{2} \mathrm{O}\right)
\end{aligned}
$$

can be used to estimate the $\mathrm{FeO} /\left(\mathrm{FeO}+\mathrm{Fe}_{2} \mathrm{O}_{3}\right)$ ratio from which the $\mathrm{Fe}_{2} \mathrm{O}_{3} / \mathrm{FeO}$ ratio can be calculated. For example, if $\mathrm{FeO} /\left(\mathrm{FeO}+\mathrm{Fe}_{2} \mathrm{O}_{3}\right)=$ $0.869565, \mathrm{Fe}_{2} \mathrm{O}_{3} / \mathrm{FeO}=(1 / 0.869565)-1=0.15$. According to the method of Middlemost (1989), the $\mathrm{Fe}_{2} \mathrm{O}_{3} / \mathrm{FeO}$ ratio is fixed based on the rock type represented by the analysis. Verma et al (2002) superpose these values on the modified total alkali vs. silica (TAS) diagram (after Le Bas et al 1986; Le Bas 1989, 2000) which can be conveniently referred to for determining the $\mathrm{Fe}_{2} \mathrm{O}_{3} / \mathrm{FeO}$ ratio. Note that for using the Middlemost method it is required to recalculate the analysis on a volatile free $100 \%$ total basis.

Once the $\mathrm{Fe}_{2} \mathrm{O}_{3} / \mathrm{FeO}$ ratio is fixed according to the method of either Le Maitre (1986) or of Middlemost (1989), the $\mathrm{FeO}$ and $\mathrm{Fe}_{2} \mathrm{O}_{3}$ amounts in the analysis can be adjusted as follows. Convert all $\mathrm{FeO}$ to $\mathrm{Fe}_{2} \mathrm{O}_{3}$ as $\mathrm{Fe}_{2} \mathrm{O}_{3}=\mathrm{FeO} \times 1.111348$ and add this amount to the already existing $\mathrm{Fe}_{2} \mathrm{O}_{3}$, if any. If $\mathrm{Fe}_{2} \mathrm{O}_{3} / \mathrm{FeO}=\mathrm{R}$,

$$
\mathrm{FeO}_{\text {Adjusted }}=\left(\frac{\left(\mathrm{Fe}_{2} \mathrm{O}_{3}\right)_{\text {Total }}}{1+\mathrm{R} \times 0.899809}\right) \times 0.899809
$$

and

$$
\left(\mathrm{Fe}_{2} \mathrm{O}_{3}\right)_{\text {Adjusted }}=\left(\mathrm{Fe}_{2} \mathrm{O}_{3}\right)_{\text {Total }}-\left(\frac{\left(\mathrm{Fe}_{2} \mathrm{O}_{3}\right)_{\text {Total }}}{1+\mathrm{R} \times 0.899809}\right) .
$$

Most up-to-date atomic weights of elements recommended by the IUPAC Commission on Atomic weights and Isotopic Abundances (Vocke 1999) have been used for the calculation of molecular weights of the oxides and the formula weights of the normative phases. For obtaining standard norms, this is important. Two calculation schemes, although being perfectly correct in their respective methodologies, will yield different norms just because they may be using different sets of atomic weights. Because SINCLAS uses the same atomic weights, it was possible to compare the results obtained from the present scheme with that obtained from SINCLAS. The present scheme failed to calculate in case of 12 analyses out of the approximately 11,200 analyses used to check its efficacy. In these analyses $1 / 3\left(\right.$ moles $\mathrm{P}_{2} \mathrm{O}_{5}$ ) exceeded $1 / 10$ (moles $\mathrm{CaO}$ ). Because all $\mathrm{P}_{2} \mathrm{O}_{5}$ is assigned to apatite, $\mathrm{CaO}$ falls short in these analyses. Such inconsistencies in the input data should be removed before the calculation of the norm is attempted.

\section{Conclusions}

Present study suggests a set of simple equations for the calculation of CIPW norms for igneous rocks. These equations are obtained by simultaneous solution by matrix methods of a set of equations representing either the breakdown of the $\mathrm{Al}_{2} \mathrm{O}_{3}$-saturated minerals to their $\mathrm{Al}_{2} \mathrm{O}_{3}$ undersaturated counterparts or that of the $\mathrm{SiO}_{2}{ }^{-}$ saturated minerals to their $\mathrm{SiO}_{2}$-undersaturated counterparts. The use of these equations for the calculation of the CIPW norm is implemented through 25 programmer-friendly steps. Use of modified molecular weights for $\mathrm{CaO}$ and $\mathrm{FeO}$, corrected formula weights for apatite and pyrite, and measures taken to avoid significant rounding off errors at step number 20 achieve complete removal of mass discrepancies between the totals of the oxides and the totals of the normative minerals. A database consisting of a worldwide compilation of about 11,200 analyses from a large number of publications that were compiled and periodically updated by the following workers: Pal et al (1978); Aguilar-Y-Vargas and Verma (1987); Verma (2000, 2002, 2004, 2006); Agrawal et al (2004, 2008); Verma et al (2006) and Agrawal and Verma (2007) provided representative 
rock chemistry data for an objective evaluation of the functioning of the algorithm presented here. See these papers for more complete list of references. The results completely agree except for apatite, as far as the norms are concerned, with the results obtained from the most recent, precise and accurate computer program SINCLAS of Verma et al (2002, 2003). Additionally, an absolute match between the totals of the oxides and the norms, which eluded all earlier schemes of norm calculation, is achieved in the present study.

\section{Acknowledgements}

I am immensely grateful to Prof. S P Verma, who reviewed the manuscript, for his constructive comments and helpful attitude. He generously supplied the database of rock analyses for evaluating the methods described in this paper and also a copy of SINCLAS for comparison of the results.

\section{References}

Agrawal S, Guevara M and Verma S P 2004 Discriminant analysis applied to establish major element field boundaries for tectonic varieties of basic rocks; Int. Geol. Rev. $46575-594$.

Agrawal S, Guevara M and Verma S P 2008 Tectonic discrimination of basic and ultrabasic volcanic rocks through log-transformed ratios of immobile trace elements; Int. Geol. Rev. 50 DOI: 10.2747/0020-6814.50.12.1.

Agrawal S and Verma S P 2007 Comment on "Tectonic classification of basalts with classification trees" by Pieter Vermeesch (2006); Geochim. Cosmochim. Acta 71 3388-3390.

Aguilar-Y-Vargas V H and Verma S P 1987 Composición química (elementos mayores) de los magmas en el Cinturón Volcánico Mexicano; Geofísica Internacional 26 195-272.

Carmichael I S E, Nicholls J and Smith A L 1970 Silica activity in igneous rocks; American Mineralogist $\mathbf{5 5}$ 246-263.

Cox K G, Bell J D and Pankhurst R J 1979 The interpretation of igneous rocks; London: Alan and Unwin, $450 \mathrm{pp}$.

Cross W, Iddings J P, Pirrson L V and Washington $\mathrm{H} \mathrm{S}$ 1902 A quantitative chemico-mineralogical classification and nomenclature of igneous rocks; J. Geol. 10 555-590.

Hutchison C S 1974 Laboratory Handbook of Petrographic Techniques (New York: John Wiley \& Sons) 527 pp.

Johannsen A 1931 A descriptive petrography of the igneous rocks; vol. 1; Chicago University Press, 267 pp.

Kelsey C H 1965 Calculation of the CIPW norm; Mineralogical Magazine 34 276-282.

Le Bas M J, Le Maitre R W, Streckeisen A and Zanettin B 1986 A chemical classification of volcanic rocks on the total alkali-silica diagram; J. Petrol. 27 $745-750$

Le Bas M J 1989 Nephelenitic and basanitic rocks; J. Petrol. 30 1299-1312.

Le Bas M J 2000 IUGS classification of the high-Mg and picritic volcanic rocks; J. Petrol. 41 1467-1470.

Le Maitre R W 1976 Some problems of the projection of chemical data into mineralogical classifications; Contrib. Mineral. Petrol. 56 181-189.

Le Maitre R W 1982 Numerical petrology: statistical interpretation of geochemical data (Amsterdam: Elsevier) $282 \mathrm{pp}$.

Middlemost E A K 1989 Iron oxidation ratios, norms and the classification of volcanic rocks; Chemical Geology $\mathbf{7 7}$ 19-26.

Pal S, López M M, Pérez R J and Terrell D J 1978 Magma characterization of the Mexican Volcanic Belt (Mexico); Bulletin Volcanologique 41 379-389.

Petrelli M, Poli G, Perugini D and Peccerillo A 2005 PetroGraph: A new software to visualize, model, and present geochemical data in igneous petrology; Geochemistry Geophysics Geosystem 6 Q07011.

Ragland P C 1989 Basic analytical petrology (Oxford: Oxford University Press) 369 pp.

Rollinson H R 1993 Using geochemical data: evaluation, presentation, interpretation (Essex: Longman) $352 \mathrm{pp}$.

Verma S P 2000 Geochemistry of the subducting Cocos plate and the origin of subduction-unrelated mafic magmas at the volcanic front of the central part of the Mexican Volcanic Belt; In: Geological Society of America Special Paper on Cenozoic Tectonics and Volcanism of Mexico, Delgado-Granados H, Aguirre-Díaz G and Stock J M (eds) No. 334, Chapter 13, 195-222.

Verma S P 2002 Absence of Cocos plate subduction-related basic volcanism in southern Mexico: A unique case on Earth?; Geology 30 1095-1098.

Verma S P 2004 Solely extension-related origin of the eastern to west-central Mexican Volcanic Belt (Mexico) from partial melting inversion model; Curr. Sci. 86 713-719.

Verma S P 2006 Extension-related origin of magmas from a garnet-bearing source in the Los Tuxtlas volcanic field, Mexico; Int. J. Earth Sci. (Geologische Rundschau) 95 871-901.

Verma S P, Guevara M and Agrawal S 2006 Discriminating four tectonic settings: Five new geochemical diagrams for basic and ultrabasic volcanic rocks based on log-ratio transformation of major-element data; J. Earth Syst. Sci. $115485-528$.

Verma S P, Torres-Alvarado I S and Sotelo-Rodríguez Z T 2002 SINCLAS: Standard igneous norm and volcanic rock classification system; Computers $\mathcal{G}$ Geosciences $\mathbf{2 8}$ $711-715$.

Verma S P, Torres-Alvarado I S and Velasco-Tapia F T 2003 A revised CIPW norm; Schweizerische Mineralogische und Petrographische Mitteilungen 83 197-216.

Vocke Jr R D 1999 IUPAC Commission on Atomic Weights and Isotopic Abundances - Atomic Weights of the Elements 1997; Pure Appl. Chemistry 71 1593-1607.

Washington H S 1917 Chemical analyses of igneous rocks; US Geological Survey Professional Paper 99.

Zhou J and Li X 2006 GeoPlot: An excel VBA program for geochemical data plotting; Computers $\&$ Geosciences 32 $554-560$. 